OPEN ACCESS

Edited by:

Xihui Shen,

Northwest $A$ and F University, China

Reviewed by:

Biswajit Maiti,

Nitte University, India

Tianpei Huang,

Fujian Agriculture and Forestry

University, China

${ }^{*}$ Correspondence:

Xuezhi Ding

dingxuezhi@hunnu.edu.cn

Specialty section:

This article was submitted to

Bacteria and Host,

a section of the journal

Frontiers in Cellular and

Infection Microbiology

Received: 15 November 2021 Accepted: 03 January 2022

Published: 25 January 2022

Citation:

Zhou P, Chen W, Zhu Z, Zhou K, Luo S, Hu S, Xia L and Ding X (2022) Comparative Study of Bacillus amyloliquefaciens X030 on the Intestinal Flora and Antibacterial Activity Against Aeromonas of Grass Carp. Front. Cell. Infect. Microbiol. 12:815436.

doi: 10.3389/fcimb.2022.815436

\section{Comparative Study of Bacillus amyloliquefaciens X030 on the Intestinal Flora and Antibacterial Activity Against Aeromonas of Grass Carp}

\author{
Pengji Zhou, Wenhui Chen, Zirong Zhu, Kexuan Zhou, Sisi Luo, Shengbiao Hu, \\ Liqiu Xia and Xuezhi Ding*
}

State Key Laboratory of Developmental Biology of Freshwater Fish, Hunan Provincial Key Laboratory of Microbial Molecular Biology, College of Life Science, Hunan Normal University, Changsha, China

Beneficial microorganisms to control bacterial diseases has been widely used in aquaculture, Bacillus amyloliquefaciens (BaX030) as a probiotic feed additive was a commonly biological control method. Added sucrose promoted the growth of BaX030, and the yield of its antibacterial substance macrolactin A was enhanced by 1.46-fold. A total of 2055 proteins were screened through proteomics, with 143 upregulated and 307 downregulated. Differential protein expression analysis and qRT-PCR verification showed that the pentose phosphate pathway and the fatty acid synthesis pathway were upregulated, thereby providing sufficient energy and precursors for the synthesis of macrolactin A. The influence of some potential regulatory factors (SecG, Lial, MecG and ComG) on macrolactin A was discovered. After grass carp were fed with BaX030, the abundance of probiotics (Fusobacterium, Proteobacteria, Gemmobacter) were higher than the control group, and the abundance of potential pathogenic bacteria (Planctomycetes, Aeromonas) were significantly lower than the control group. The cell and challenge experiments showed that BaX030 can significantly increase the expression of $C 3$ and IL8 in the liver and kidney, which decreases the risk of immune organ disease. Moreover, BaX030 effectively reduced the mortality of grass carp. The results revealed that $\mathrm{BaX030}$ can significantly improve the structure of the intestinal flora, enhance immunity and it is beneficial to the control of grass carp Aeromonas.

Keywords: Bacillus amyloliquefaciens, aquaculture disease, proteomics, intestinal flora, grass carp

\section{HIGHLIGHTS}

- BaX030 changed the intestinal flora, including Fusobacterium, Proteobacteria and Planctomycetes

- Sucrose affected the growth and the macrolactin A synthesis of BaX030

- BaX030 can improve the specific immunity in grass carp 


\section{INTRODUCTION}

The increasing density of fish farming and the deterioration of the aquatic environment have led to disease outbreaks and aquaculture losses. The traditional extensive use of antibiotics will lead to environmental pollution, pathogen resistance and drug residues in aquatic products, which can seriously threaten human health (Mukherjee et al., 2019; Zhi et al., 2020). Therefore, improving the microbial community by probiotic supplementation has been suggested as promising approach in the prevention of diseases. Bacillus amyloliquefaciens can produce a large number of secondary metabolites with a wide range of antibacterial activities, including polyketide compounds (bacillaene, macrolactin and difficidin) and lipopeptide compounds (surfactins, iturins and fengycins) (Debois et al., 2014; Cochrane and Vederas, 2016). As an effective strategy of biological control, it has great potential in aquaculture.

At present, systematically mining multiple core genes and proteins has become a research trend; it explains the molecular mechanism underlying the synthesis of secondary metabolites and the increase of their production through genomics combined with transcriptomics, metabolomics or proteomics (Chavali and Rhee, 2018; Xu et al., 2018; Zhao et al., 2018). Proteomics has produced a variety of novel leads for the analysis of protein traffic in Bacillus. These studies proved that the availability of genomics, proteomics and bioinformatics tools can effectively increase the yield of secondary metabolites and modify related gene clusters.

The intestine of grass carp is the organ with the largest and the most complex microbial flora. The gut microbiota is colonized by diverse probiotics and pathogenic microbes (Pérez et al., 2010; Zhou et al., 2019), and it is involved in many physiological processes of the host and plays crucial roles, such as enhancing host metabolism, nutrient absorption, growth and disease resistance. (Rooks and Garrett, 2016; Blander et al., 2017; Huang et al., 2018; Rimoldi et al., 2018); therefore, it has been widely studied worldwide. However, the intestine can also become an important entry point for bacteria to invade grass carp. Under artificial breeding conditions, grass carp are easily infected with bacterial diseases and die, which causes huge economic losses (Song et al., 2014). For example, bacterial sepsis is caused by Aeromonas hydrophila (Elbehiry et al., 2019), skin ulcers, and severe ascites are caused by Aeromonas veronii (Li et al., 2020), and these species often colonize the intestine to exert pathogenicity. Enteric inflammation is another important factor that leads to the high mortality of grass carp (Tran et al., 2018) because of the disorder it causes to the structure, function, and metabolism of the microbial flora. Carding et al. (2015) found that enteric pathogens trigger both local and systemic inflammation that changes the composition of microbiota and barrier functions. Therefore, studying the intestinal microbiota and probiotics has great significance for the prevention and treatment of intestinal diseases. The latest third-generation sequencing (TGS) is characterized by single molecules and long reads, which may enable high-throughput full-length $16 \mathrm{~S}$ sequencing. TGS has reached a level of species identification accuracy similar to that of first-generation sequencing and the breadth of application similar to that of the next-generation sequencing (NGS), and it is expected to be widely used in the study of intestinal flora in the future (Van Dijk et al., 2018; Kumar et al., 2019).

We discovered the effect of sucrose on bacterial growth and macrolactin A production. B.amyloliquefaciens X030 (BaX030) provided immune protection to grass carp against infection by $A$. hydrophila and $A$. veronii in vivo and in vitro. Differences in the intestinal microbiota between diseased and healthy grass carp were investigated by TGS technology. Our results are important in the prevention and treatment of intestinal diseases by controlling gut microbes.

\section{MATERIALS AND METHODS}

\section{Bacterial Strains and Culture Conditions}

BaX030 was screened and isolated from soil in Henan Province, China. It is currently deposited in the Wuhan Center for Culture Collection (CCTCC NO: M2014159), and the whole-genome sequence data are available in the GenBank database under the accession number CP040672.1. Cao et al. (2019) and Huang et al. (2020) isolated A. hydrophila X040 (AhX040) (GenBank: KU159281.1) and A. veronii X005 (AvX005) (GenBank: KU641116.1) from diseased grass carp on a Wangcheng fish farm (Changsha, Hunan Province, China), respectively. They were common bacteria in freshwater, sewage, silt and soil, and ther were also the main pathogenic bacteria that caused diseases in freshwater farmed. The LB medium contained $10 \mathrm{~g} / \mathrm{L} \mathrm{NaCl}, 10$ $\mathrm{g} / \mathrm{L}$ tryptone, and $5 \mathrm{~g} / \mathrm{L}$ yeast extract. The fermentation medium consisted of LB medium supplemented with $32 \mathrm{~g} / \mathrm{L}$ sucrose.

After BaX030 was activated overnight, it was transferred to LB medium or fermentation medium at a ratio of $3 \%$ and named $\mathrm{CG}+\mathrm{BaX} 030$ and $\mathrm{SG}+\mathrm{BaX} 030$, respectively. Three replicates were performed for each group. The fermentation broth was diluted to a certain concentration every $4 \mathrm{~h}$, and the $\mathrm{OD}_{600 \mathrm{~nm}}$ value was measured with a SmartSpecTM 3000 spectrophotometer (BioRad, Berkeley, USA). A growth curve was drawn with the culture time at the abscissa and the $\mathrm{OD}_{600 \mathrm{~nm}}$ value at the ordinate. Small amounts of fermentation broth were taken at different time points during growth, centrifuged at $10000 \mathrm{rpm}$ for $2 \mathrm{~min}$ to collect the bacteria. An AXIO Observer A1 upright optical microscope (Zeiss, Jena, Germany) was used for observation.

\section{Separation and Purification of Antibacterial Active Substances}

BaX030 was cultured on the LB medium and the fermentation medium for $48 \mathrm{~h}$, and its fermentation broth was centrifuged at $10000 \mathrm{rpm}$ for $10 \mathrm{~min}$. The supernatant was added to an equal volume of ethyl acetate overnight extraction, and the upper organic phase was freeze-dried, dissolved in 100\% methanol and analysed by high-performance liquid chromatography (HPLC 1290, Agilent, Palo Alto, USA). The injection volume was $10 \mu \mathrm{L}$, the column was a reversed-phase ZORBAX SB-C18 column $(4.6 \times 150 \mathrm{~mm}, 5.0 \mu \mathrm{m})$, and the flow rate was $1 \mathrm{~mL} / \mathrm{min}$, with mobile phases A consisting of $10 \%$ acetonitrile (ACN) and 
B consisting of $90 \%$ ACN. Mobile phase B was gradient eluted from $100 \%$ ( $0 \mathrm{~min}$ ) to $0 \%$ (20 $\mathrm{min})$, and the detection wavelength was $280 \mathrm{~nm}$. After collecting and freeze-drying the elution peaks, antibacterial experiments were used to detect the activity of the peak by the filter paper method with AvX005 and AhX040 as indicator bacteria. The activity peak was polyketide macrolactin A (Zhou et al., 2021). The concentration of macrolactin A (the standard curve was drawn after quantification by HPLC 1290) was determined using the following formula: macrolactin A: $\mathrm{y}=1801.1 \mathrm{x}+1.23\left(\mathrm{R}^{2}=0.9989\right)$ where $\mathrm{y}$ is the peak area and $\mathrm{x}$ is the macrolactin A concentration.

\section{Whole-Cell Protein Extraction and SDS-PAGE}

We extracted the whole protein of BaX030 at $20 \mathrm{~h}$, the protein concentration was determined by a quantitative proteomics kit (Sangon Biotech, Shanghai, China) and microplate reader (Spectral Max M5, Silicon Valley, USA). The microplate reader was used to measure the absorbance at $595 \mathrm{~nm}$, and the protein concentration in the sample was calculated according to the standard curve. Both BSA standards and samples were repeated twice. SDS-PAGE detected whether the protein was degraded, the band analysis was performed with Gel-Pro Analyzer 4.0 software. The samples were finally sent to Shanghai Medical College of Fudan University for isobaric tags for relative and absolute quantification (iTRAQ).

\section{Proteolysis and iTRAQ}

One tube of iTRAQ reagent was added per $100 \mu \mathrm{g}$ peptide, incubated at room temperature for $2 \mathrm{~h}$, mixed with $50 \mu \mathrm{L}$ ultrapure water and left at room temperature for $30 \mathrm{~min}$; each group of labelled products was combined in one tube and then dried by vacuum concentrator. The peptide samples were reconstituted in loading buffer of ultra performance liquid chromatography (UPLC), and it was performed on a Waters ACQUITY UPLC by using a reversed-phase C18 column (1.7 $\mu \mathrm{m}, 3 \mathrm{~mm} \times 150 \mathrm{~mm}$, Waters, Milford, USA) (Bekker-Jensen et al., 2017; Hu et al., 2019). According to the BaX030 genome annotation, the SwissProt/UniProt database was used for protein identification. The critical range of fold change $(F C)<0.667$ and FC $>1.50$ was used to screen different proteins $(P<0.05)$. Data are available via ProteomeXchange with identifier PXD024498 (Reviewer account details: Username: reviewer_pxd024498@ ebi.ac.uk; Password: etGXrDFP).

\section{BaX030 on the Immune Protection of Grass Carp}

We took 120 healthy grass carp (weight $4.6 \pm 2.0 \mathrm{~g}$, body length $6.5 \pm 1.0 \mathrm{~cm}$ ), and kept them in $30 \mathrm{~L}$ fish tanks, the water volume of each fish tank was $20 \mathrm{~L}$. This was two groups (fed without BaX030, control group, and fed with BaX030, experimental group) in triplicates. BaX030 was counted by the plate colony counting method, and the amount of bacteria mixed into the feed was $1 \times 10^{9}$ colony forming units/gram (CFU/g). It was cultured in fermentation medium for $20 \mathrm{~h}, 1.5 \mathrm{~mL}$ of bacterial liquid was mixed with $1 \mathrm{~g}$ of feed, and the bacterial content of the feed was
$1 \times 10^{9} \mathrm{CFU} / \mathrm{g}$. The daily feed amount was $3 \%$ of the fish's weight given twice a day. After all fish were fed for 30 days, 3 fish were randomly taken from the WT group and the BA group, their intestinal contents were collected for TGS. We took 6 other fish and intraperitoneally injected them with $0.1 \mathrm{~mL}$ of $1 \times 10^{6}$ colony forming units/milliliter (CFU/mL) AhX040 or $0.15 \mathrm{~mL}$ of $1 \times 10^{9}$ $\mathrm{CFU} / \mathrm{mL}$ AvX005. After $12 \mathrm{~h}$, the fish were dissected for collection of the liver and kidney. Quantitative real-time polymerase chain reaction (qRT-PCR) was used to analyze the expression of immune genes. Pathological characteristics were observed through H\&E staining. The blank control was injected with the same dose of PBS. The concentration of BaX030 was determined by co-cultivation experiments in vitro.

Co-cultivation test: AhX040 and BaX030 were cultured overnight. The concentration of AhX040 was adjusted to $1 \times 10^{6}$ $\mathrm{CFU} / \mathrm{mL}$ and the concentration of BaX030 was adjusted to $1 \times 10^{9}$ $\mathrm{CFU} / \mathrm{g}$ with fermentation medium. Three groups were set up in the experiment: $1 \times 10^{9} \mathrm{CFU} / \mathrm{g} \mathrm{BaX030}$ was inoculated into the fermentation medium; $1 \times 10^{6} \mathrm{CFU} / \mathrm{mL}$ AhX040 was inoculated into the fermentation medium; $1 \times 10^{6} \mathrm{CFU} / \mathrm{mL}$ AhX040 and $1 \times 10^{9} \mathrm{CFU} / \mathrm{g}$ BaX030 were simultaneously inoculated into the fermentation medium, and the two bacteria were mixed culture. We took $1 \mathrm{~mL}$ of bacterial solution every $4 \mathrm{~h}$, spread the plate to count, and drew the growth curve of the co-culture of AhX040 and BaX030. The determination of AvX005 was consistent with the above method.

\section{Genomic DNA Extraction and Sequencing}

Extraction of total bacterial DNA from intestinal samples was used for high-throughput sequencing in grass carp, the samples were tested by a Bioanalyzer system 2100 (Agilent, Palo Alto, USA) and then subjected to sequencing by a PacBio platform and data analysis (Wagner et al., 2016).

\section{Bioinformatics Analysis}

Raw reads were filtered to remove adaptors and low-quality and ambiguous bases, and then paired-end reads were added to tags by the Fast Length Adjustment of Short reads program (FLASH, v1.2.11) (Magoc and Salzberg, 2011) to obtain the tags. The tags were clustered into operational taxonomic units (OTUs) with a cut-off value of 97\% using UPARSE software (v7.0.1090) (Edgar, 2013), and chimaera sequences were compared with the Gold database using UCHIME (v4.2.40) (Edgar et al., 2011). Then, OTU representative sequences were taxonomically classified using Ribosomal Database Project (RDP) Classifier v.2.2 with a minimum confidence threshold of 0.6 and trained on the GreenGenes database v201305 by QIIME v1.8.0 (Caporaso et al., 2010). USEARCH_global (Edgar, 2010) was used to compare all tags with the OTUs to obtain the OTU abundance statistics table for each sample. Alpha and beta diversity was estimated by MOTHUR (v1.31.2) (Schloss et al., 2009) and QIIME at the OTU level, respectively. The Venn plots of the OTUs and OTU rank curves were plotted with VennDiagram version 3.1.1. Sample clustering was conducted by QIIME based on UPGMA. LEfSe cluster was conducted by LEfSe. KEGG analysis was performed using PICRUSt software 
(Wilkinson et al., 2018). Barplots and heat maps of different classification levels were plotted with $\mathrm{R}$ package.

\section{RNA Extraction and qRT-PCR}

BaX030 was cultured in the two media for $20 \mathrm{~h}$ and centrifuged at $10000 \mathrm{rpm}$ for $5 \mathrm{~min}$ to collect approximately $0.1 \mathrm{~g}$ of bacteria, and the total RNA of BaX030 was extracted by Trizol method (Yi et al., 2018). The RNA concentration was determined by NanoDrop 2000 spectrophotometer (Thermo Scientific, Waltham, USA), and the reverse transcription kit of the PrimeScriptTM RT reagent Kit with gDNA Eraser (TaKaRa, Dalian, China) was used to perform genomic DNA removal and reverse transcription reactions. The relative expression level of each mRNA was normalized to $16 \mathrm{~S}$ rRNA as the internal reference, The transcription levels of seven genes, leuA, $z w f$, $l e u D, \sec G, p d k 2$, pckA and gerPC, were detected.

To evaluate the mRNA expression level of fish liver and kidney, we selected the housekeeping gene $\beta$-actin as the reference gene (Wang et al., 2015; Yang et al., 2016) and selected 5 immunerelated genes, immunoglobulin $\mathrm{M}(\mathrm{IgM})$, complement $\mathrm{C} 3$ (C3),

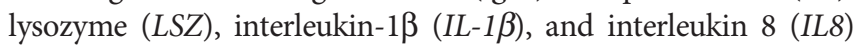
(Supplementary Table S1). All primers were synthesized by Sangon Biotech (Shanghai, China) and Primer Premier version 5 (Premier Biosoft International, Palo Alto, USA) was used for primer design.

\section{qRT-PCR}

The reaction was carried out on an ABI 7500 real-time PCR system (Applied Biosystems, Waltham, USA). The total reaction system was $20 \mu \mathrm{L}$ in eight tubes: $10 \mu \mathrm{L}$ of SYBRTM Green Mix (Applied Biosystems, Waltham, USA), $7.8 \mu \mathrm{L}$ of ultrapure water, $0.6 \mu \mathrm{L}$ of F primer, $0.6 \mu \mathrm{L}$ of $\mathrm{R}$ primer, and $1 \mu \mathrm{L}$ of $\mathrm{cDNA}$. The reaction programme was $50^{\circ} \mathrm{C}$ for $2 \mathrm{~min}$; predenaturation at $95^{\circ} \mathrm{C}$ for $10 \mathrm{~min}$; and then a total of $40 \mathrm{cycles}$ of $95^{\circ} \mathrm{C}$ of denaturation for $30 \mathrm{~s}$ and $60^{\circ}$ $\mathrm{C}$ for $1 \mathrm{~min}$ for annealing. Finally, the $2^{-\Delta \Delta \mathrm{Ct}}$ relative quantitative method was used to compare and analyse the expression levels of gene mRNA in the samples. Each sample was repeated 4 times.

\section{Protection Experiment of BaX030}

A total of 225 healthy grass carp were divided into the feeding group $(\mathrm{BA}+\mathrm{Ah}, \mathrm{BA}+\mathrm{Av})$ and the control group $(\mathrm{WT}+\mathrm{Ah}, \mathrm{WT}+$ $\mathrm{Av}, \mathrm{WT}+\mathrm{PBS})$. The water temperature was controlled at $25^{\circ} \mathrm{C}$, and the volume of each fish tank was $20 \mathrm{~L}$. The feeding group was cultured with the BaX030 strain with fermentation medium, mixed into the feed at $1 \times 10^{9} \mathrm{CFU} / \mathrm{g}$ and fed twice a day. After thirty days, 45 fish from each group were injected with the same concentration of AhX040 or AvX005 and observed for seven days. Their cumulative mortality was calculated. The protective effect of the BaX030 strain on grass carp was evaluated according to the relative percentage survival rate $(\mathrm{RPS}=(1$-feeding group mortality rate/positive control mortality rate) $\times 100 \%)$. The same amount of PBS was injected as a blank control group.

\section{Cytotoxicity Test of Macrolactin A on Grass Carp Liver Cells}

We added $100 \mu \mathrm{L}\left(1 \times 10^{4}\right.$ cells $\left./ \mathrm{mL}\right)$ of L8824 liver cells to the wells of a 96-well plate and cultured them in a cell incubator held consistently at $30^{\circ} \mathrm{C}$ for $12 \mathrm{~h} .10 \mu \mathrm{L}$ of $20 \mu \mathrm{M}$ macrolactin A was added to the wells of the plate. The fermentation supernatants of AhX040 and AvX005 were used as controls. The cells were cultured for $48 \mathrm{~h}$, and the cell morphology was observed under an inverted microscope (DMIL, Mannheim, Germany) at 20-times magnification.

\section{RESULTS}

\section{Growth Curve Determination and Phase Contrast Microscope Observation}

The addition of sucrose promoted cell growth (Figure 1A). CG+ $\mathrm{BaX} 030$ and $\mathrm{SG}+\mathrm{BaX} 030$ grew slowly in the lag phase (0-4 h); the bacteria grew rapidly in the log phase (4-16 h). After $16 \mathrm{~h}$, the amount of bacteria remained stable, then began to decline after approximately $32 \mathrm{~h}$ of incubation. $\mathrm{CG}+\mathrm{BaX} 030$ began to produce spores at $20 \mathrm{~h}$, Sporulation was delayed by 12 hours in the experimental group (Supplementary Figure S1). The addition of sucrose also promoted the biosynthesis of secondary metabolites,
A

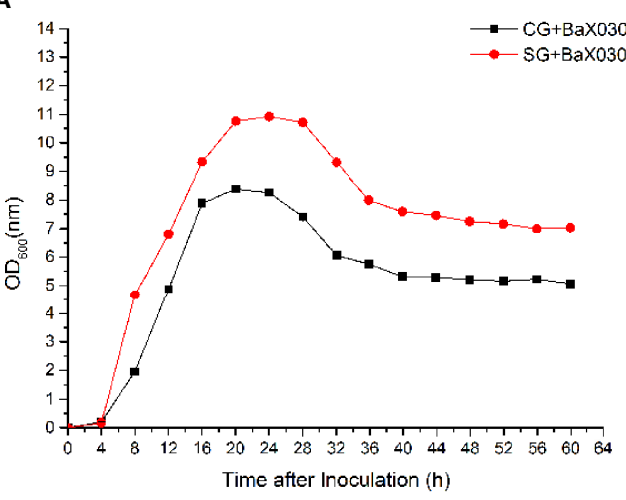

B

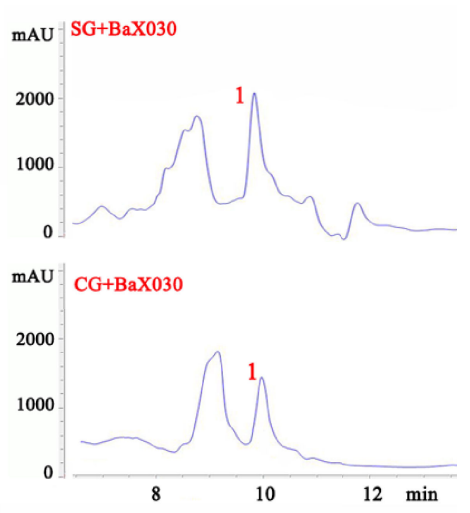

FIGURE 1 | Growth parameters and separation and purification of BaX030. (A) The growth curve of CG+BaX030 and SG+BaX030. Datas were mean \pm SEM for $\mathrm{n}=3$ biologically independent experiments; (B) HPLC chromatograms of BaX030 in different media. 
and the peak area corresponding to polyketide macrolactin A was increased (Figure 1B).

\section{Yield of Macrolactin A and pH Changes}

According to the yield curve, the production of macrolactin A had the same obvious upward trend in the early stage, especially in the logarithmic phase (Figure 2A). The yield of SG+BaX030 was significantly increased from $8.12 \mu \mathrm{g} / \mathrm{mL}$ to $11.84 \mu \mathrm{g} / \mathrm{mL}$ compared with CG at $48 \mathrm{~h}$. During cultivation, the $\mathrm{pH}$ of the experimental group first dropped to 5.02 and then increased, while the control group was basically in an upward trend. The $\mathrm{pH}$ level had an observable antibacterial effect on AhX040 and AvX005 at $20 \mathrm{~h}$ (Figure 2B).

\section{Statistical Analysis of Differential Proteins}

Whole protein was extracted from BaX030 in the LB medium and the fermentation medium, and SDS-PAGE showed clear bands (Supplementary Figure S2). The total number of proteins was identified as 2055 by iTRAQ, including 143 upregulated proteins and 307 downregulated proteins (Figure 3A). Gene

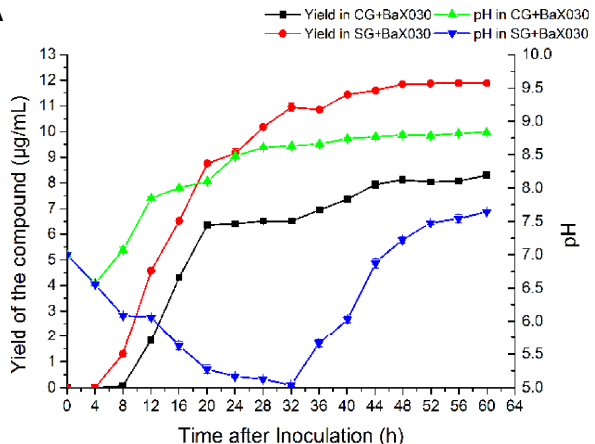

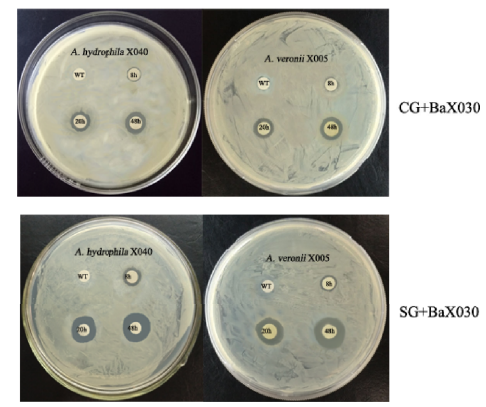

FIGURE 2 | The yield change graph and antibacterial of macrolactin A. (A) The yield curve of macrolactin A in two media. Datas were mean \pm SEM for $n=3$ biologically independent experiments; (B) The antibacterial experiment of fermentation broth on AhX040 and AvX005 at different time points WT: Original medium without inoculation.

A

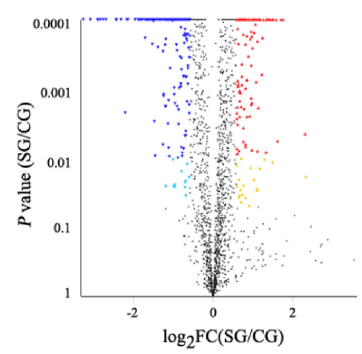

C

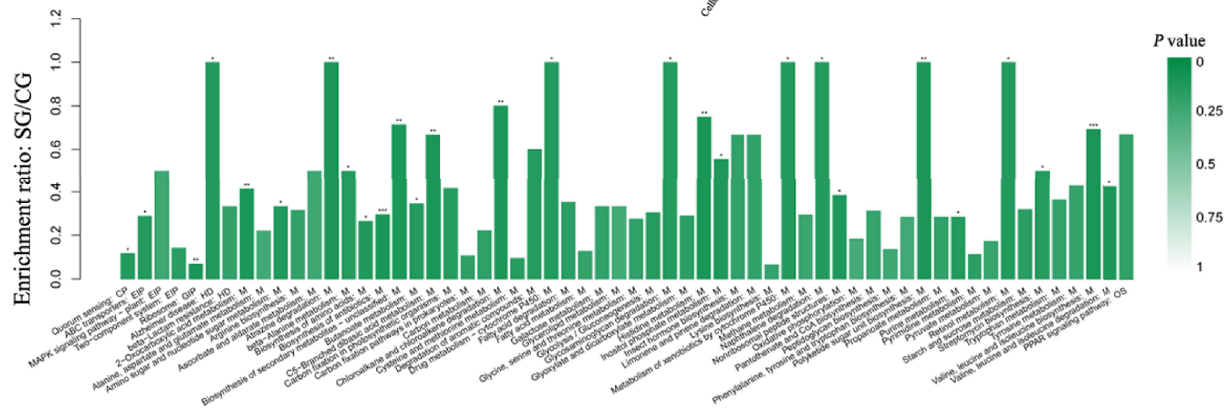

B

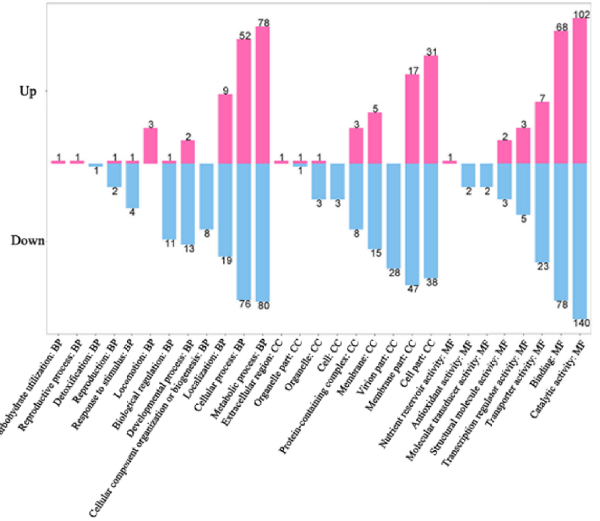

differential proteins; (C) KEGG analysis, biological pathways involved in differential proteins. ${ }^{\star \star \star} P<0.001 ;{ }^{\star \star} P<0.01 ;{ }^{\star} P<0.05$. 
Ontology (GO) analysis found that compared to CG+BaX030, the proteins of $\mathrm{SG}+\mathrm{BaX} 030$ were significantly enriched in the molecular function (MF) process of catalytic activity. The second was the biological process (BP) of metabolism (Figure 3B).

\section{Analysis the Differential Proteins by KEGG Pathway}

The metabolic pathways of 450 differentially expressed proteins were classified by Kyoto Encyclopedia of Genes and Genomes (KEGG). We found that the most different proteins were involved in metabolic processes (M) (Figure 3C). We detected the upregulated genes $f b a A$, $g a p A$, and $g p m I$ in the glycolysis pathway and the downregulated gene pepck in the gluconeogenesis pathway (Figure 4) (Supplementary Table S2), which contributed to the enrichment of pyruvate and acetyl-CoA. They provided an important raw material for the synthesis of fatty acids, and the significant increase in macrolactin A was largely dependent on the accumulation of fatty acids. A large number of proteins were upregulated in the pentose phosphate and the tricarboxylic acid cycle pathway, which supplied cells with sufficient carbon scaffolds and ATP.

\section{Newly Discovered Potential Functional Proteins That Importantly Impact the Expression of Macrolactin A}

According to proteomic data, the expression of the $p k s$ operon was increased after adding sucrose (gene cluster 9) (Figure 5), so we searched for proteins related to macrolactin A to further evaluate their potential impact, including several newly discovered proteins. The anti-sigma $\mathrm{F}$ factor encoded by spoIIAB was upregulated, which was involved in the formation of spores in stage II. It inhibited the release of the precursor-sigma $\mathrm{F}$ factor $\left(\right.$ pro- $\left.\sigma^{F}\right)$ and led to the downregulation of RNA polymerase- $\sigma^{E}\left(E-\sigma^{E}\right)$. LiaI is a small membrane protein with two transmembrane helices that may be able to counteract antibiotic-induced damage, which is beneficial to the production of metabolites. AspP showed the same expression trend as macrolactin $\mathrm{A}$, which proved its positive effect on macrolactin A. The preprotein translocase subunit SecG promoted the ATP-dependent translocation of precursor PhoB (Pro-phoB) and then affected macrolactin A.

\section{qRT-PCR Verification}

The three bands of $23 \mathrm{~S}, 16 \mathrm{~S}$ and $5 \mathrm{~S}$ rRNA were clear (Supplementary Figure S3), and the quality of the extracted RNA was good. The $\mathrm{qRT}-\mathrm{PCR}$ results showed that the transcription fold increases of leuA, zwf, leuD, and secG were 3.61, 2.07, 2.96 and 5.06 times, respectively. The transcription fold values of $p d k 2, p c k A$ and gerPC were $0.48,0.43$ and 0.31 times, respectively, which was also consistent with the same trend observed in the proteomics data (Supplementary Figure S4; Supplementary Table S2).

\section{Analysis of the Structural Characteristics of the Intestinal Microbiome}

The Venn diagram showed that the total number of OTUs was 245 in the two groups, and the unique OTU numbers of the EX group



FIGURE 4 | The diagram of metabolic pathways may be affected by addition of sucrose for BaX030, including glycolysis/gluconeogenesis, TCA cycle, pentose phosphate pathway, purine metabolism, fatty acid/amino acid synthesis and metabolism, etc. The marked genes in red corresponding to the proteins were upregulated, and the green were down-regulated. 


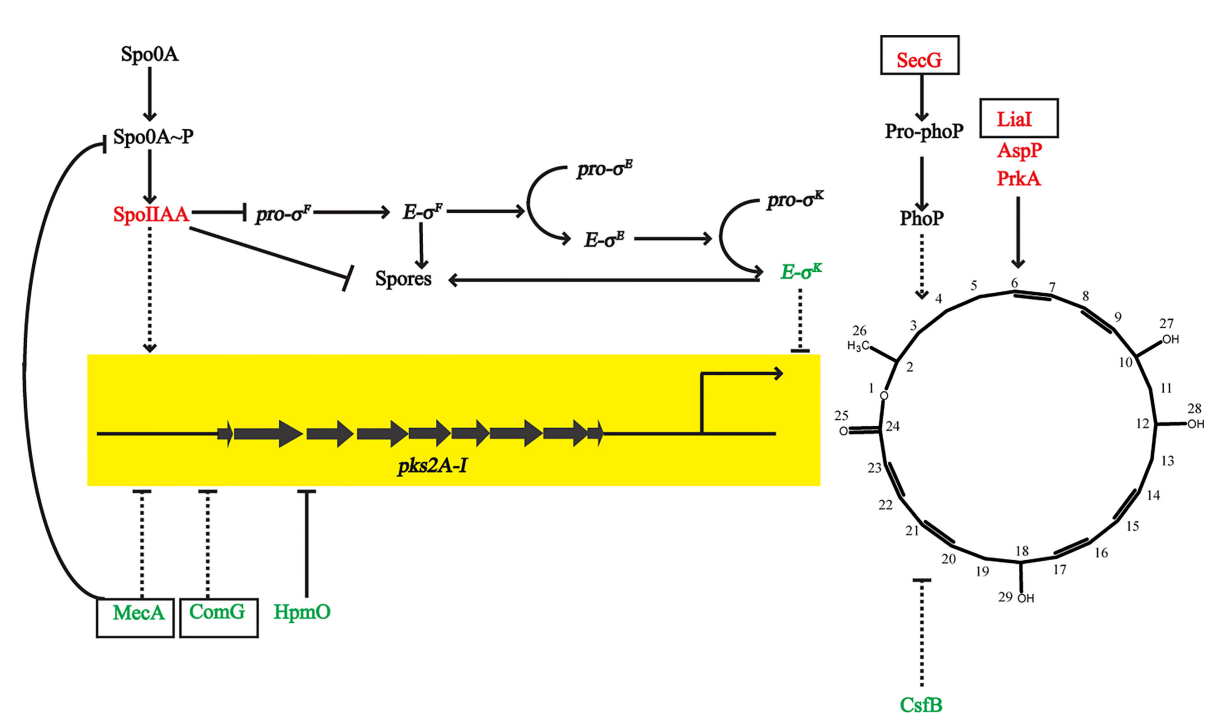

FIGURE 5 | Differential proteins that affected the expression of macrolactin A. Arrows and T-shapes indicated positive and negative regulation, respectively. The solid indicate direct effect, and the dotted line indicate indirect effect. The black box marks represented new discovered and important proteins in this study.

and the WT group were 27 and 37, respectively (Figure 6A), which indicated that $\mathrm{BaX} 030$ can change the composition of the intestinal flora of grass carp. An $\alpha$ diversity analysis showed that the coverage of intestinal samples exceeded $99 \%$ in the EX and WT groups. The observed species index, Chao index, Ace index, and Shannon index of the EX group were lower than those of the WT group, and the Simpson index was greater than that of the WT group. The OTU rank curve showed that the experimental group had a faster downward trend than the control group. The results demonstrated that $\mathrm{BaX} 030$ can increase the proportion of the dominant flora and reduce the diversity of the community in the gut microbiome (Figure 6B, Supplementary Figure S5). We found that the experimental group and the control group were clustered on their respective branches by clustering trees, their composition structure was different (Figure 6C).

\section{Analysis of the Abundance of Intestinal Microbiome}

A total of 10 bacterial phyla were identified from in 6 samples. Proteobacteria, Planctomycetes, Actinobacteria, Fusobacteria,
A

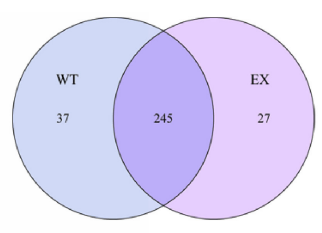

c unweighted_unifrac

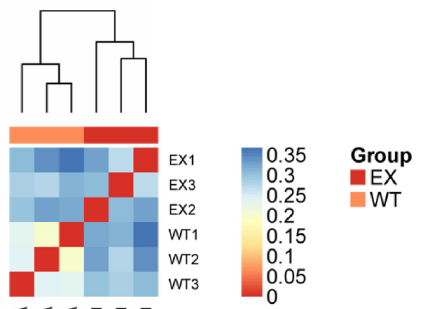

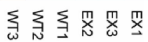

B
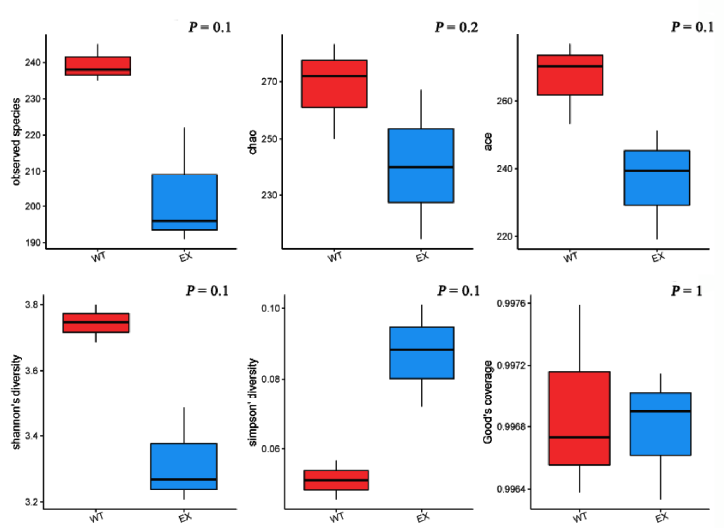

FIGURE 6 | The structural characteristics of the intestinal microbiome. (A) Venn diagram of two groups intestinal samples of BaX030-added or not based on OTU; (B) Diversity analysis of the intestinal microbiota in grass carp between healthy and diseased groups; (C) The heat map of Beta diversity. 
Verrucomicrobia, Bacteroidetes, Firmicutes and Chloroflexiare were phyla with an abundance of more than $0.1 \%$. Proteobacteria $(55.48 \pm$ $18.46 \%)$ and Planctomycetes $(25.32 \pm 8.44 \%)$ dominated the taxonomic composition in the EX and WT (45.24 $\pm 3.38 \%$ and $36.04 \pm 5.09 \%$, respectively) groups (Figure 7A). The control group showed a relatively high average abundance of Actinobacteria $(9.80 \% \pm$ $0.49 \%$ ), whereas the treatment group showed a relative dominance of Fusobacteria $(9.27 \pm 7.96 \%)$. Additionally, the pathogens Aeromonas were reduced from $5.2 \% \pm 1.32 \%$ to $3.37 \pm 1.19 \%$.

LEfSe was performed to characterize the microbial communities exhibiting significant differences in abundance between the WT and EX groups. The results showed that the relative abundances of the families Rhodobacteraceae, Nocardioidaceae, Geobacteraceae and Ignavibacteriaceae were significantly higher in the EX group, while the relative abundances of the families Planctomycetaceae, Actinomycetaceae, Verrucomicrobiaceae, and Enterobacteriaceae were significantly increased in the WT group $(P<0.05)$ (Figure 7B), which suggested that metabolic changes occurred in the intestinal microbiota of grass carp caused by the feeding of BaX030.

\section{Influence of BaX030 on Specific Immunity in Grass Carp}

The expression of immune-related genes in the liver and kidney was determined by qRT-PCR (Supplementary Figure S6). In the liver, the gene expression levels of $I g M$ and $I L 8$ in the BA+Ah group were significantly higher than those in the $\mathrm{BA}+\mathrm{Av}$ and control groups, and the expression levels of $C 3$ were significantly improved in the two groups (Figure 8). In the kidney, the expression level of $\operatorname{IgM}$ was higher in the $\mathrm{BA}+\mathrm{Ah}$ group than in the other groups. However, the upregulation of the IL8 gene was lower than that in the $\mathrm{BA}+\mathrm{Av}$ group. The expression level of $I L-1 \beta$ was increased only in the $\mathrm{BA}+\mathrm{Av}$ group.

\section{Pathological Observation of Grass Carp}

The WT+Ah group began to show signs of stress at $12 \mathrm{~h}$, including obvious congestion of the abdomen and pelvic fins, red and swollen anus, and scale loss. The $\mathrm{WT}+\mathrm{Av}$ group mainly manifested in slight congestion of the abdomen and pelvic fins and red and swollen anus at $48 \mathrm{~h}$. However, the symptoms were relieved in the experimental group (Supplementary Figure S7).

$\mathrm{H} \& \mathrm{E}$ staining results showed that the hepatocytes of grass carp infected with AhX040 were exfoliated by apoptosis and separated from the outer membrane of blood vessels (Figure 9); the nephrocytes were severely vacuolated, denatured and necroti, the intercellular space was enlarged. The vascular cells infected with AvX005 showed vacuolar degeneration and separation from surrounding tissues. A large number of cells were exfoliated to form large cavitie. The corresponding pathological signs were improved in the experimental group. The results showed that BaX030 could protect the immune organs and reduce the virulence of the pathogen.

\section{Protection Experiment of BaX030}

The fermentation supernatant of the $\mathrm{SG}+\mathrm{BaX} 030$ group was not toxic to liver cells, as indicated in cell experiments. The cells were
A

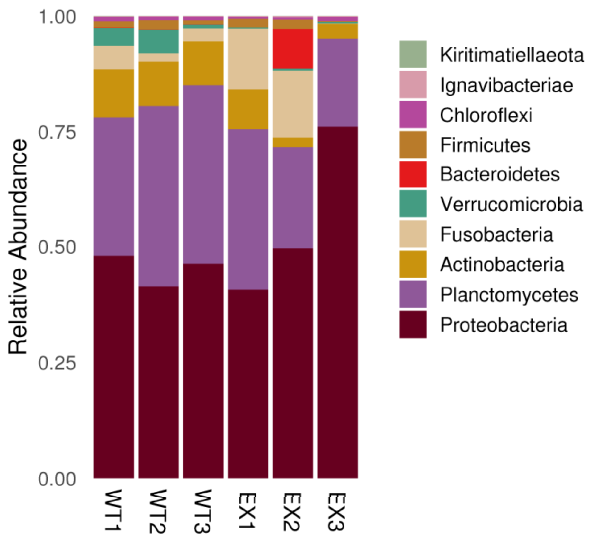



FIGURE 7 | Effects of BaX030 on the intestinal flora of grass carp. (A) The composition of bacterial community on total 6 samples at phylum level; (B) Effect of $\mathrm{BaX030}$ on the intestinal microbiome of grass carp by LefSe analysis. 
A

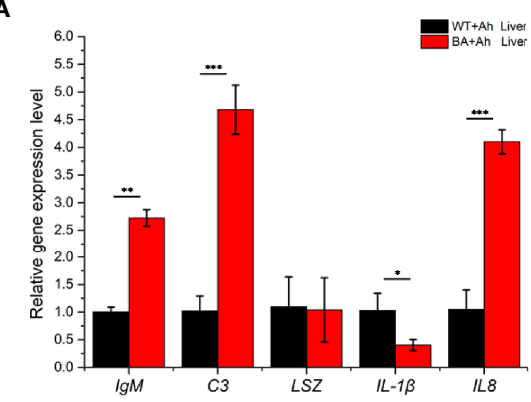

C

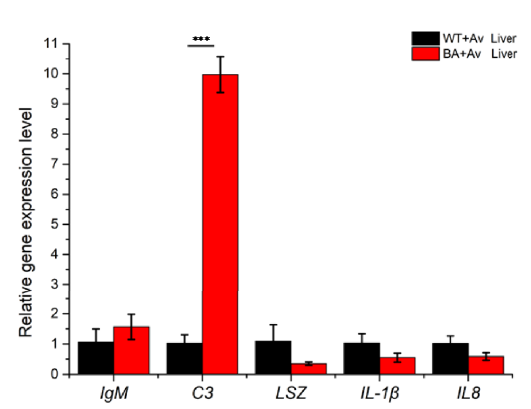

B



D

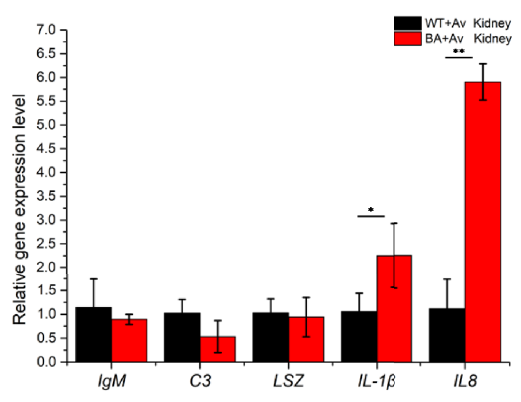

FIGURE 8 | Analysis of immune-related gene expression in grass carp that fed with BaX030 after infection with AhX040 and AvX005. (A, B) Expression of immunerelated genes in the liver and kidney after infection with AhX040; (C, D) The gene expression levels of immune-related cytokines in liver and kidney at post-infection AvX005. WT+Ah: grass carp+AhX040; BA+Ah: grass carp+BaX030+AhX040; WT+Av: grass carp+AvX005; BA+Av: grass carp+BaX030+AvX005. Datas were mean \pm SEM for $n=4$ biologically independent experiments. Statistical analysis were performed using one-way ANOVA. ${ }^{\star \star \star} P<0.001 ;{ }^{\star \star} P<0.01 ;{ }^{\star} P<0.05$.

contracted and round, which proved that they all had died in the Ah group and the Av group. In the macrolactin A group, the cell arrangement was loose, and the number of cells was increased compared to the control, which had a certain protective effect on cells (Supplementary Figure S8).

When the initial dose of $1 \times 10^{9} \mathrm{CFU} / \mathrm{g} \mathrm{BaX} 030$ was co-cultured with $1 \times 10^{6} \mathrm{CFU} / \mathrm{mL}$ AhX040 or $1 \times 10^{9} \mathrm{CFU} / \mathrm{mL}$ AvX005, BaX030 could completely inhibit the growth of two pathogens (Supplementary Figure S9). After intraperitoneal injection of AhX040 and AvX005, grass carp were continuously observed for 4 and 9 days, respectively, and the cumulative survival rate was determined. The results showed that the cumulative survival rate of the BA+Ah group was $20.0 \%$, which was $13.3 \%$ lower than that of the control group, and the RPS value was $14.3 \%$. The cumulative survival rate of the $\mathrm{BA}+\mathrm{Av}$ group was $51.1 \%$, which was $22.2 \%$ lower than that of the control group, and the RPS value was $37.1 \%$ (Figure 10). Therefore, BaX030 can reduce the damage caused by two pathogens in grass carp.

\section{DISCUSSION}

Many antibiotics had stimulating activity at low concentrations, which is called "hormesis". They induced transcriptional activation and promoted the growth and biofilm formation of Bacillus (Hoffman et al., 2005; Davies et al., 2006). Sucrose stimulated bacterial growth, which led to an increase in the production of macrolactin A (Figures 1A, 2A). Macrolactin A may regulate its own "hormesis" effect by inducing bacteria to produce certain signalling molecules when irritated by sucrose (Liu et al., 2018). The total production of bacteria and macrolactin A was higher than that of the control group. We tried to add other carbon sources, including glucose, fructose, maltose, lactose, and starch, but the effect was not as good as that of sucrose. BaX030 was the low utilization of some carbon sources due to different bacterial growth characteristics, such as maltose, lactose, etc. Another possible reason was that although other carbon sources were added to promote the growth of the strain, it did not lead to the increase in the yield of the target product macrolactin A. Sucrose additional was an effective strategy because it is a cheaper carbon source and readly available in nature. B. amyloliquefaciens has higher sucrose hydrolysis activity, a lower free sugar consumption rate, and can use sucrose to grow rapidly, resulting in a higher biomass yield (Bhatia et al., 2018). In addition, polyketides often need to carry other functional groups to have strong biological activity on the carbon atom framework, such as hydroxyl groups, carboxyl groups, double bonds, amino acid methylation, or glycoside formation. Macrolactin A constitutes a class of 24membered macrolide compounds, and it had a good antibacterial effect because its structure contained a large number of carboncarbon double bonds and hydroxyl groups (Ortiz and Sansinenea, 2020).

A. hydrophila and A. veronii can adhere to the intestinal tract and at the same time produce toxins to make fish sick (Wang et al., 2016; Zhang et al., 2019; Zhang et al., 2020). Our results 




FIGURE 9 | The pathological changes of the liver and kidney in grass carp were observed by H\&E staining (400x). The black arrow indicated the hepatocytes were exfoliated by apoptosis, the blue arrow indicated the nephrocyte were exfoliated to form large cavities.

showed that pathological damage mainly included damage to the liver, kidney and other immune organs, and the expression of related immune genes was downregulated, which ultimately reduced the resistance of grass carp to pathogen invasion (Figures 8, 9). B. amyloliquefaciens can regulate fish growth and immunity by colonizing fish intestines, where it can compete with pathogenic microorganisms for adhesion sites and inhibit the proliferation of Aeromonas species because of its high rate of adhesion to the intestinal mucosa (Thankappan et al., 2015; Santos et al., 2021). Many studies have proven that Bacillus can produce antibacterial active substances and reduce mortality in aquaculture (Pinchuk et al., 2001; Nayak, 2010). Therefore, Bacillus can colonize in fish intestines and can also produce


we considered increasing the amount of the strain applied or cotreating fish with other probiotics as a means of improving the resistance of the fish to pathogenic bacteria.
The dominant phyla contained Proteobacteria, Firmicutes, Bacteroidetes and Actinobacteria in the experimental group and the control group. As the core microbiota of the intestine in grass carp, these bacteria can effectively regulate the structure of the intestinal microbiome and affect its healthy growth (Han et al., 2010; Roeselers et al., 2011; Yang et al., 2019). These bacteria contain a diverse functional repertoire, including chemoorganotrophic ability to utilize environment-derived fatty acids, aromatics, carbohydrates, and peptides (Zhou et al., 2020). Because of these abilities Proteobacteria in high abundance indirectly promoted the adaptability of grass carp to the environment. Moreover, Proteobacteria and Actinobacteria also had a certain positive effect on the production of polyketides, including macrolactin A (Desriac et al., 2013; Buijs et al., 2019). Li et al. (2013) found that the relative abundance of Firmicutes and Bacteroides affects the growth of fish; the ratio was higher, and the growth rate was faster. The ratio was decreased after the grass carp were fed BaX030, but the two groups 




FIGURE 10 | The cumulative survival rate of grass carp in control group and experimental group. (A) The cumulative survival rate of grass carp after being infected with AhX040; (B) The cumulative survival rate of grass carp after being infected with AvX005.

were not different in terms of body weight. Therefore, the validity of this potential marker is worth discussing. Currently, it is difficult to relate the ratio of Firmicutes/Bacteroidetes to the growth status in grass carp (Magne et al., 2020). In addition, the abundance of probiotic Gemmobacter in the experimental group (16.9\%) was higher than that in the control group (10.4\%), and the abundance of potential pathogens, such as Aeromonas, was lower than that in the control group, which indicated that the intestinal microbiome of grass carp fed BaX030 had a better structure and a healthier composition.

The interaction between probiotic bacilli and fish immune cells can lead to an enhanced immune response (Sangma and Kamilya, 2015; Gobi et al., 2018). Previous reports have proven that adding $B$. amyloliquefaciens to feed can enhance the immunity and disease resistance of Nile tilapia, Labeo rohita and zebrafish against $A$. hydrophila infection (Nandi et al., 2018; Lin et al., 2019; Kuebutornye et al., 2020). IL-1 $\beta$ and IL8 are the two main proinflammatory cytokines in fish, and they can balance the inflammatory response to bacterial infection (Reis et al., 2012; Wang et al., 2019). The biological activity of IL8 can attract and activate neutrophils. After contacting $I L 8$, neutrophils will undergo morphological changes, move towards the reaction site, and release a series of active products (Wang et al., 2017). C3 had the highest degree of upregulation in the liver, which proved that $B$. amyloliquefaciens can potentially improve liver stress tolerance (Singh et al., 2017). The C3 and IL8 levels of the liver and kidney of grass carp were significantly increased in the experimental group, indicating that the upregulation of two immune factors was essential for the inhibition of AhX040 and AvX005. LSZ is a small-molecule alkaline enzyme that hydrolyses mucopolysaccharides and can block the invasion of foreign pathogenic bacteria (Derde et al., 2017). IgM is the immunoglobulin with the largest molecular weight and can lyse bacteria and neutralize viruses. Its deficiency lead to a tendency for sepsis and/or pulmonary oedema caused by gram-negative bacteria to develop (Bilal et al., 2016; Su et al., 2018). This finding was consistent with our results; BaX030 was beneficial to the improvement of immune factor $\operatorname{IgM}$ and reduced the pathological sign in grass carp.

When the prkA gene was destroyed, the activity of $B$. amyloliquefaciens was reduced (Liu et al., 2013), so the significantly upregulation of PrkA may have promoted the production of secondary metabolites. The ComGA-G operon of the binding and transport of DNA (Briley et al., 2011) in Bacillus and the two-component stimulating factor CsfB ( $\mathrm{Lu}$ et al., 2019) with quorum sensing activity showed the opposite expression trend of macrolactin A, which suggested their negative effects on its production. The expression of HPMO can degrade hexadecane and cause fatty acids to not be converted into polyketides efficiently, which was not conducive to the expression of the pks operon (Yu et al., 2013). The adapter protein MecA targeted the transcription factor ComK and was then degraded by the ClpC/ ClpP proteolytic complex to negatively regulate the activities of Bacillus, including directly binding and inhibiting the transcriptional activity of Spo0A-P, as well as inhibiting the biofilm formation activity (Prepiak et al., 2011). It was worth mentioning that we also detected the corresponding up-regulation of NRPS in the proteomics data, especially involving the synthesis of fengycins (Supplementary Table S2). However, it may not have a direct antibacterial effect through using an agar diffusion test, so we probably removed it in the separation and purification. Instead, it competed with certain sites in the quorum sensing system for A.hydrophila and A.veronii decolonization (Piewngam et al., 2018). The mechanism was interesting and can be studied further.

In conclusion, the study revealed that sucrose improved the growth of BaX030, affected key functional proteins and potential regulators of macrolactin $\mathrm{A}$, and enhanced the antibacterial activity. It provided a detailed database of key primary metabolism, secondary metabolism and regulatory proteins related to biosynthesis. The yield of target products can be further improved by optimizing the culture medium and genetic modification in the industry. Protection experiments showed that $\mathrm{BaX} 030$ was not only beneficial to the intestinal flora in grass carp, but also modulated the immune responses and function against AhX040 and AvX005 infections. The probiotic Bacillus has potential application value as a feed additive and good prospects for its application in aquaculture, immunity, and control of fish diseases. Our study laid a foundation for establishing the relationship between economic traits and intestinal microbiota. 


\section{DATA AVAILABILITY STATEMENT}

The datasets presented in this study can be found in online repositories. The names of the repository/repositories and accession number(s) can be found in the article/Supplementary Material.

\section{ETHICS STATEMENT}

This study had been reviewed and approved by the Ethics Committee of Hunan Normal University, the approval number was 2021388 .

\section{AUTHOR CONTRIBUTIONS}

$\mathrm{PZ}$ wrote the manuscript. WC and $\mathrm{ZZ}$ conducted the experiments. $\mathrm{KZ}$ and SL participated in sample processing. $\mathrm{SH}$

\section{REFERENCES}

Bekker-Jensen, D. B., Kelstrup, C. D., Batth, T. S., Larsen, S. C., Haldrup, C., Bramsen, J. B., et al. (2017). An Optimized Shotgun Strategy for the Rapid Generation of Comprehensive Human Proteomes. Cell Syst. 4 (6), 587-599.e4. doi: 10.1016/j.cels.2017.05.009

Bhatia, S. K., Yoon, J. J., Kim, H. J., Hong, J. W., Gi Hong, Y., Song, H. S., et al. (2018). Engineering of Artificial Microbial Consortia of Ralstonia Eutropha and Bacillus Subtilis for Poly(3-Hydroxybutyrate-Co-3-Hydroxyvalerate) Copolymer Production From Sugarcane Sugar Without Precursor Feeding. Bioresour. Technol. 257, 92-101. doi: 10.1016/j.biortech.2018.02.056

Bilal, S., Lie, K. K., Karlsen, O. A., and Hordvik, I. (2016). Characterization of IgM in Norwegian Cleaner Fish (Lumpfish and Wrasses). Fish Shellfish Immunol. 59, 9-17. doi: 10.1016/j.fsi.2016.09.063

Blander, J. M., Longman, R. S., Iliev, I. D., Sonnenberg, G. F., and Artis, D. (2017). Regulation of Inflammation by Microbiota Interactions With the Host. Nat. Immunol. 18 (8), 851-860. doi: 10.1038/ni.3780

Briley, K.Jr, Dorsey-Oresto, A., Prepiak, P., Dias, M. J., Mann, J. M., and Dubnau, D. (2011). The Secretion ATPase ComGA is Required for the Binding and Transport of Transforming DNA. Mol. Microbiol. 81 (3), 818-830. doi: 10.1111/j.1365-2958.2011.07730.x

Buijs, Y., Bech, P. K., Vazquez-Albacete, D., Bentzon-Tilia, M., Sonnenschein, E. C., Gram, L., et al. (2019). Marine Proteobacteria as a Source of Natural Products: Advances in Molecular Tools and Strategies. Nat. Prod. Rep. 36 (9), 1333-1350. doi: 10.1039/c9np00020h

Cao, L., Pan, L., Gong, L., Yang, Y., He, H., Li, Y., et al. (2019). Interaction of a Novel Bacillus Velezensis (BvL03) Against Aeromonas Hydrophila In Vitro and In Vivo in Grass Carp. Appl. Microbiol. Biotechnol. 103 (21-22), 8987-8999. doi: 10.1007/s00253-019-10096-7

Caporaso, J. G., Kuczynski, J., Stombaugh, J., Bittinger, K., Bushman, F. D., Costello, E. K., et al. (2010). QIIME Allows Analysis of High-Throughput Community Sequencing Data. Nat. Methods 7 (5), 335-336. doi: 10.1038/ nmeth.f.303

Carding, S., Verbeke, K., Vipond, D. T., Corfe, B. M., and Owen, L. J. (2015). Dysbiosis of the Gut Microbiota in Disease. Microb. Ecol. Health Dis. 26, 26191. doi: 10.3402/mehd.v26.26191

Chavali, A. K., and Rhee, S. Y. (2018). Bioinformatics Tools for the Identification of Gene Clusters That Biosynthesize Specialized Metabolites. Brief Bioinform. 19 (5), 1022-1034. doi: 10.1093/bib/bbx020

Cochrane, S. A., and Vederas, J. C. (2016). Lipopeptides From Bacillus and Paenibacillus Spp.: A Gold Mine of Antibiotic Candidates. Med. Res. Rev. 36 (1), 4-31. doi: 10.1002/med.21321

Davies, J., Spiegelman, G. B., and Yim, G. (2006). The World of Subinhibitory Antibiotic Concentrations. Curr. Opin. Microbiol. 9 (5), 445-453. doi: 10.1016/ j.mib.2006.08.006 and LX analyzed data. XD conceived the research and completed the revision of the manuscript. All authors read and approved the manuscript.

\section{FUNDING}

This work was supported by the National key Research and Development program of China (2017YFD0201201), the National Natural Science Foundation of China (31370116).

\section{SUPPLEMENTARY MATERIAL}

The Supplementary Material for this article can be found online at: https://www.frontiersin.org/articles/10.3389/fcimb.2022.815436/ full\#supplementary-material

Debois, D., Jourdan, E., Smargiasso, N., Thonart, P., De Pauw, E., and Ongena, M. (2014). Spatiotemporal Monitoring of the Antibiome Secreted by Bacillus Biofilms on Plant Roots Using MALDI Mass Spectrometry Imaging. Anal. Chem. 86 (9), 4431-4438. doi: 10.1021/ac500290s

Derde, M., Vié, V., Walrant, A., Sagan, S., Lechevalier, V., Guérin-Dubiard, C., et al. (2017). Antimicrobial Activity of Lysozyme Isoforms: Key Molecular Features. Biopolymers 107 (12), e23040. doi: 10.1002/bip.23040

Desriac, F., Jégou, C., Balnois, E., Brillet, B., Le Chevalier, P., and Fleury, Y. (2013). Antimicrobial Peptides From Marine Proteobacteria. Mar. Drugs 11 (10), 3632-3660. doi: 10.3390/md11103632

Edgar, R. C. (2010). Search and Clustering Orders of Magnitude Faster Than BLAST. Bioinformatics 26 (19), 2460-2461. doi: 10.1093/bioinformatics/ btq461

Edgar, R. C. (2013). UPARSE: Highly Accurate OTU Sequences From Microbial Amplicon Reads. Nat. Methods 10 (10), 996-998. doi: 10.1038/nmeth.2604

Edgar, R. C., Haas, B. J., Clemente, J. C., Quince, C., and Knight, R. (2011). UCHIME Improves Sensitivity and Speed of Chimera Detection. Bioinformatics 27 (16), 2194-2200. doi: 10.1093/bioinformatics/btr381

Elbehiry, A., Marzouk, E., Abdeen, E., Al-Dubaib, M., Alsayeqh, A., Ibrahem, M., et al. (2019). Proteomic Characterization and Discrimination of Aeromonas Species Recovered From Meat and Water Samples With a Spotlight on the Antimicrobial Resistance of Aeromonas Hydrophila. Microbiologyopen 8 (11), e782. doi: 10.1002/mbo3.782

Gobi, N., Vaseeharan, B., Chen, J. C., Rekha, R., Vijayakumar, S., Anjugam, M., et al. (2018). Dietary Supplementation of Probiotic Bacillus Licheniformis Dahb1 Improves Growth Performance, Mucus and Serum Immune Parameters, Antioxidant Enzyme Activity as Well as Resistance Against Aeromonas Hydrophila in Tilapia Oreochromis Mossambicus. Fish Shellfish Immunol. 74, 501-508. doi: 10.1016/j.fsi.2017.12.066

Han, S., Liu, Y., Zhou, Z., He, S., Cao, Y., Shi, P., et al. (2010). Analysis of Bacterial Diversity in the Intestine of Grass Carp (Ctenopharyngodon Idella) Based on $16 S$ rDNA Gene Sequences. Aquac. Res. 42, 47-56. doi: 10.1111/j.13652109.2010.02543.x

Hoffman, L. R., D’Argenio, D. A., MacCoss, M. J., Zhang, Z., Jones, R. A., and Miller, S. I. (2005). Aminoglycoside Antibiotics Induce Bacterial Biofilm Formation. Nature 436 (7054), 1171-1175. doi: 10.1038/nature03912

Huang, C., Wu, P., Jiang, W. D., Liu, Y., Zeng, Y. Y., Jiang, J., et al. (2018). Deoxynivalenol Decreased the Growth Performance and Impaired Intestinal Physical Barrier in Juvenile Grass Carp (Ctenopharyngodon Idella). Fish Shellfish Immunol. 80, 376-391. doi: 10.1016/j.fsi.2018.06.013

Huang, H., Zhou, P., Chen, P., Xia, L., Hu, S., Yi, G., et al. (2020). Alteration of the Gut Microbiome and Immune Factors of Grass Carp Infected With Aeromonas Veronii and Screening of an Antagonistic Bacterial Strain (Streptomyces Flavotricini). Microb. Pathog. 143, 104092. doi: 10.1016/ j.micpath.2020.104092 
Hu, H. L., Kang, Y., Zeng, Y., Zhang, M., Liao, Q., Rong, M. Q., et al. (2019). Region-Resolved Proteomics Profiling of Monkey Heart. J. Cell Physiol. 234 (8), 13720-13734. doi: $10.1002 /$ jcp.28052

Kuebutornye, F. K. A., Wang, Z., Lu, Y., Abarike, E. D., Sakyi, M. E., Li, Y., et al. (2020). Effects of Three Host-Associated Bacillus Species on Mucosal Immunity and Gut Health of Nile Tilapia, Oreochromis Niloticus and its Resistance Against Aeromonas Hydrophila Infection. Fish Shellfish Immunol. 97, 83-95. doi: 10.1016/j.fsi.2019.12.046

Kumar, K. R., Cowley, M. J., and Davis, R. L. (2019). Next-Generation Sequencing and Emerging Technologies. Semin. Thromb. Hemost. 45 (7), 661-673. doi: 10.1055/s-0039-1688446

Lin, Y. S., Saputra, F., Chen, Y. C., and Hu, S. Y. (2019). Dietary Administration of Bacillus Amyloliquefaciens R8 Reduces Hepatic Oxidative Stress and Enhances Nutrient Metabolism and Immunity Against Aeromonas Hydrophila and Streptococcus Agalactiae in Zebrafish (Danio Rerio). Fish Shellfish Immunol. 86, 410-419. doi: 10.1016/j.fsi.2018.11.047

Li, T., Raza, S. H. A., Yang, B., Sun, Y., Wang, G., Sun, W., et al. (2020). Aeromonas Veronii Infection in Commercial Freshwater Fish: A Potential Threat to Public Health. Anim. (Basel) 10 (4), 608. doi: 10.3390/ani10040608

Liu, Z., Budiharjo, A., Wang, P., Shi, H., Fang, J., Borriss, R., et al. (2013). The Highly Modified Microcin Peptide Plantazolicin is Associated With Nematicidal Activity of Bacillus Amyloliquefaciens FZB42. Appl. Microbiol. Biotechnol. 97 (23), 10081-10090. doi: 10.1007/s00253-013-5247-5

Liu, Y., Kyle, S., and Straight, P. D. (2018). Antibiotic Stimulation of a Bacillus Subtilis Migratory Response. mSphere 3 (1), e00586-e00517. doi: 10.1128/ mSphere.00586-17

Li, X. M., Yan, Q. Y., Xie, S. Q., Hu, W., Yu, Y. H., and Hu, Z. H. (2013). Gut Microbiota Contributes to the Growth of Fast-Growing Transgenic Common Carp (Cyprinus Carpio L.). PloS One 8 (5), e64577. doi: 10.1371/ journal.pone.0064577

Lu, J. Y., Zhou, K., Huang, W. T., Zhou, P., Yang, S., Zhao, X., et al. (2019). A Comprehensive Genomic and Growth Proteomic Analysis of Antitumor Lipopeptide Bacillomycin Lb Biosynthesis in Bacillus Amyloliquefaciens X030. Appl. Microbiol. Biotechnol. 103 (18), 7647-7662. doi: 10.1007/s00253019-10019-6

Magne, F., Gotteland, M., Gauthier, L., Zazueta, A., Pesoa, S., Navarrete, P., et al. (2020). The Firmicutes/Bacteroidetes Ratio: A Relevant Marker of Gut Dysbiosis in Obese Patients? Nutrients 12 (5):1474. doi: 10.3390/nu12051474

Magoc, T., and Salzberg, S. L. (2011). FLASH: Fast Length Adjustment of Short Reads to Improve Genome Assemblies. Bioinformatics 27 (21), 2957-2963. doi: 10.1093/bioinformatics/btr507

Mukherjee, A., Banerjee, G., Mukherjee, P., Ray, A. K., Chandra, G., and Ghosh, K. (2019). Antibacterial Substances Produced by Pathogen Inhibitory Gut Bacteria in Labeo Rohita: Physico-Chemical Characterization, Purification and Identification Through MALDI-TOF Mass Spectrometry. Microb. Pathog. 130, 146-155. doi: 10.1016/j.micpath.2019.02.028

Nandi, A., Banerjee, G., Dan, S. K., Ghosh, K., and Ray, A. K. (2018). Evaluation of In Vivo Probiotic Efficiency of Bacillus Amyloliquefaciens in Labeo Rohita Challenged by Pathogenic Strain of Aeromonas Hydrophila MTCC 1739. Probiotics Antimicrob. Proteins 10 (2), 391-398. doi: 10.1007/s12602-0179310-x

Nayak, S. K. (2010). Probiotics and Immunity: A Fish Perspective. Fish Shellfish Immunol. 29 (1), 2-14. doi: 10.1016/j.fsi.2010.02.017

Ortiz, A., and Sansinenea, E. (2020). Macrolactin Antibiotics: Amazing Natural Products. Mini Rev. Med. Chem. 20 (7), 584-600. doi: 10.2174/ 1389557519666191205124050

Pérez, T., Balcázar, J., Ruiz-Zarzuela, I., Halaihel, N., Vendrell, D., De Blas, I., et al. (2010). Host-Microbiota Interactions Within the Fish Intestinal Ecosystem. Mucosal Immunol. 3 (4), 355-360. doi: 10.1038/mi.2010.12

Piewngam, P., Zheng, Y., Nguyen, T. H., Dickey, S. W., Joo, H. S., Villaruz, A. E., et al. (2018). Pathogen Elimination by Probiotic Bacillus via Signalling Interference. Nature 562 (7728), 532-537. doi: 10.1038/s41586-018-0616-y

Pinchuk, I. V., Bressollier, P., Verneuil, B., Fenet, B., Sorokulova, I. B., Megraud, F., et al. (2001). In Vitro Anti-Helicobacter Pylori Activity of the Probiotic Strain Bacillus Subtilis 3 Is Due to Secretion of Antibiotics. Antimicrob. Agents Chemother. 45 (11), 3156-3161. doi: 10.1128/AAC.45.11.3156-3161.2001

Prepiak, P., Defrancesco, M., Spadavecchia, S., Mirouze, N., Albano, M., Persuh, M., et al. (2011). MecA Dampens Transitions to Spore, Biofilm
Exopolysaccharide and Competence Expression by Two Different Mechanisms. Mol. Microbiol. 80 (4), 1014-30.x. doi: 10.1111/j.13652958.2011.07627.x

Reis, M. I., do Vale, A., Pereira, P. J., Azevedo, J. E., and Dos Santos, N. M. (2012). Caspase-1 and $I L-1 \beta$ Processing in a Teleost Fish. PloS One 7 (11), e50450. doi: 10.1371/journal.pone.0050450

Rimoldi, S., Terova, G., Ascione, C., Giannico, R., and Brambilla, F. (2018). Next Generation Sequencing for Gut Microbiome Characterization in Rainbow Trout (Oncorhynchus Mykiss) Fed Animal by-Product Meals as an Alternative to Fishmeal Protein Sources. PloS One 13 (3), e0193652. doi: 10.1371/journal.pone.0193652

Roeselers, G., Mittge, E. K., Stephens, W. Z., Parichy, D. M., Cavanaugh, C. M., Guillemin, K., et al. (2011). Evidence for a Core Gut Microbiota in the Zebrafish. ISME J. 5 (10), 1595-1608. doi: 10.1038/ismej.2011.38

Rooks, M. G., and Garrett, W. S. (2016). Gut Microbiota, Metabolites and Host Immunity. Nat. Rev. Immunol. 16 (6), 341-352. doi: 10.1038/nri.2016.42

Sangma, T., and Kamilya, D. (2015). Dietary Bacillus Subtilis FPTB13 and Chitin, Single or Combined, Modulate Systemic and Cutaneous Mucosal Immunity and Resistance of Catla, Catla Catla (Hamilton) Against Edwardsiellosis. Comp. Immunol. Microbiol. Infect. Dis. 43, 8-15. doi: 10.1016/j.cimid.2015.09.003

Santos, R. A., Oliva-Teles, A., Pousao-Ferreira, P., Jerusik, R., Saavedra, M. J., Enes, P., et al. (2021). Isolation and Characterization of Fish-Gut Bacillus Spp. As Source of Natural Antimicrobial Compounds to Fight Aquaculture Bacterial Diseases. Mar. Biotechnol. (N.Y.) 23 (2), 276-293. doi: 10.1007/s10126-02110022-x

Schloss, P. D., Westcott, S. L., Ryabin, T., Hall, J. R., Hartmann, M., Hollister, E. B., et al. (2009). Introducing Mothur: Open-Source, Platform-Independent, Community-Supported Software for Describing and Comparing Microbial Communities. Appl. Environ. Microbiol. 75 (23), 7537-7541. doi: 10.1128/ AEM.01541-09

Singh, S. T., Kamilya, D., Kheti, B., Bordoloi, B., and Parhi, J. (2017). Paraprobiotic Preparation From Bacillus Amyloliquefaciens FPTB16 Modulates Immune Response and Immune Relevant Gene Expression in Catla Catla (Hamilton 1822). Fish Shellfish Immunol. 66, 35-42. doi: 10.1016/j.fsi.2017.05.005

Song, X., Zhao, J., Bo, Y., Liu, Z., Wu, K., and Gong, C. (2014). Aeromonas Hydrophila Induces Intestinal Inflammation in Grass Carp (Ctenopharyngodon Idella): An Experimental Model. Aquaculture 434, 171178. doi: 10.1016/j.aquaculture.2014.08.015

Su, Y. L., Wang, B., Hu, M. D., Cui, Z. W., Wan, J., Bai, H., et al. (2018). SiteSpecific N-Glycan Characterization of Grass Carp Serum IgM. Front. Immunol. 9, 2645. doi: 10.3389/fimmu.2018.02645

Thankappan, B., Ramesh, D., Ramkumar, S., Natarajaseenivasan, K., and Anbarasu, K. (2015). Characterization of Bacillus Spp. From the Gastrointestinal Tract of Labeo Rohita-Towards to Identify Novel Probiotics Against Fish Pathogens. Appl. Biochem. Biotechnol. 175 (1), 340-353. doi: 10.1007/s12010-014-1270-y

Tran, N. T., Zhang, J., Xiong, F., Wang, G. T., Li, W. X., and Wu, S. G. (2018). Altered Gut Microbiota Associated With Intestinal Disease in Grass Carp (Ctenopharyngodon Idellus). World J. Microbiol. Biotechnol. 34 (6), 71. doi: 10.1007/s11274-018-2447-2

Van Dijk, E. L., Jaszczyszyn, Y., Naquin, D., and Thermes, C. (2018). The Third Revolution in Sequencing Technology. Trends Genet. 34 (9), 666-681. doi: 10.1016/j.tig.2018.05.008

Wagner, J., Coupland, P., Browne, H. P., Lawley, T. D., Francis, S. C., and Parkhill, J. (2016). Evaluation of PacBio Sequencing for Full-Length Bacterial 16S rRNA Gene Classification. BMC Microbiol. 16 (1), 274. doi: 10.1186/s12866-0160891-4

Wang, X., Ma, G., Zhang, R., Liu, L., Zhu, J., and Zhu, H. (2019). Molecular Characterization and Biological Functioning of Interleukin-8 in Siberian Sturgeon (Acipenser Baeri). Fish Shellfish Immunol. 90, 91-101. doi: 10.1016/ j.fsi.2019.04.010

Wang, Y., Ren, Z., Fu, L., and Su, X. (2016). Two Highly Adhesive Lactic Acid Bacteria Strains are Protective in Zebrafish Infected With Aeromonas Hydrophila by Evocation of Gut Mucosal Immunity. J. Appl. Microbiol. 120 (2), 441-451. doi: 10.1111/jam.13002

Wang, E., Wang, K., Chen, D., Wang, J., He, Y., Long, B., et al. (2015). Evaluation and Selection of Appropriate Reference Genes for Real-Time Quantitative PCR Analysis of Gene Expression in Nile Tilapia (Oreochromis Niloticus) During 
Vaccination and Infection. Int. J. Mol. Sci. 16 (5), 9998-10015. doi: 10.3390/ ijms 16059998

Wang, G. L., Wang, M. C., Zhang, X. W., Chang, M. X., Xie, H. X., and Nie, P. (2017). Molecular Cloning, Biological Effect, and Tissue Distribution of Interleukin-8 Protein in Mandarin Fish (Siniperca Chuasti) Upon Flavobacterium Columnare Infection. Fish Shellfish Immunol. 66, 112-119. doi: 10.1016/j.fsi.2017.05.016

Weiss, G., Kovalerchick, D., Lieman-Hurwitz, J., Murik, O., De Philippis, R., Carmeli, S., et al. (2019). Increased Algicidal Activity of Aeromonas Veronii in Response to Microcystis Aeruginosa: Interspecies Crosstalk and Secondary Metabolites Synergism. Environ. Microbiol. 21 (3), 1140-1150. doi: 10.1111/1462-2920.14561

Wilkinson, T. J., Huws, S. A., Edwards, J. E., Kingston-Smith, A. H., Siu-Ting, K., Hughes, M., et al. (2018). CowPI: A Rumen Microbiome Focussed Version of the PICRUSt Functional Inference Software. Front. Microbiol. 9, 01095. doi: $10.3389 /$ fmicb.2018.01095

Xu, B. H., Lu, Y. Q., Ye, Z. W., Zheng, Q. W., Wei, T., Lin, J. F., et al. (2018). Genomics-Guided Discovery and Structure Identification of Cyclic Lipopeptides From the Bacillus Siamensis JFL15. PloS One 13 (8), e0202893. doi: 10.1371/journal.pone.0202893

Yang, G., Jian, S. Q., Cao, H., Wen, C., Hu, B., Peng, M., et al. (2019). Changes in Microbiota Along the Intestine of Grass Carp (Ctenopharyngodon Idella): Community, Interspecific Interactions, and Functions. Aquaculture 498, 151161. doi: 10.1016/j.aquaculture.2018.08.062

Yang, Y., Yu, H., Li, H., and Wang, A. (2016). Transcriptome Profiling of Grass Carp (Ctenopharyngodon Idellus) Infected With Aeromonas Hydrophila. Fish Shellfish Immunol. 51, 329-336. doi: 10.1016/j.fsi.2016.02.035

Yi, Y., Zhang, Z., Zhao, F., Liu, H., Yu, L., Zha, J., et al. (2018). Probiotic Potential of Bacillus Velezensis JW: Antimicrobial Activity Against Fish Pathogenic Bacteria and Immune Enhancement Effects on Carassius Auratus. Fish Shellfish Immunol. 78, 322-330. doi: 10.1016/j.fsi.2018.04.055

Yu, C., Yao, J., Cai, M., Wang, F., Masakorala, K., Liu, H., et al. (2013). Functional Gene Expression of Oil-Degrading Bacteria Resistant to Hexadecane Toxicity. Chemosphere 93 (7), 1424-1429. doi: 10.1016/j.chemosphere.2013.07.035

Zhang, D. X., Kang, Y. H., Zhan, S., Zhao, Z. L., Jin, S. N., Chen, C., et al. (2019). Effect of Bacillus Velezensis on Aeromonas Veronii-Induced Intestinal Mucosal Barrier Function Damage and Inflammation in Crucian Carp (Carassius Auratus). Front. Microbiol. 10, 2663. doi: 10.3389/fmicb.2019.02663

Zhang, W., Wei, L., Xu, R., Lin, G., Xin, H., Lv, Z., et al. (2020). Evaluation of the Antibacterial Material Production in the Fermentation of Bacillus
Amyloliquefaciens-9 From Whitespotted Bamboo Shark (Chiloscyllium Plagiosum). Mar. Drugs 18 (2), 119. doi: 10.3390/md18020119

Zhao, J., Zhang, C., and Lu, Z. (2018). Differential Proteomics Research of Bacillus Amyloliquefaciens and its Genome-Shuffled Saltant for Improving Fengycin Production. Braz. J. Microbiol. 49 Suppl 1 (Suppl 1), 166-177. doi: 10.1016/ j.bjm.2018.04.010

Zhi, S., Shen, S., Zhou, J., Ding, G., and Zhang, K. (2020). Systematic Analysis of Occurrence, Density and Ecological Risks of 45 Veterinary Antibiotics: Focused on Family Livestock Farms in Erhai Lake Basin, Yunnan, China. Environ. Pollut. 267, 115539. doi: 10.1016/j.envpol.2020.115539

Zhou, P., Huang, H., Lu, J., Zhu, Z., Xie, J., Xia, L., et al. (2021). The Mutated Bacillus Amyloliquefaciens Strain Shows High Resistance to Aeromonas Hydrophila and Aeromonas Veronii in Grass Carp. Microbiol. Res. 250, 126801. doi: 10.1016/j.micres.2021.126801

Zhou, L., Lin, K. T., Gan, L., Sun, J. J., Guo, C. J., Liu, L., et al. (2019). Intestinal Microbiota of Grass Carp Fed Faba Beans: A Comparative Study. Microorganisms 7 (10):465. doi: 10.3390/microorganisms7100465

Zhou, Z., Tran, P. Q., Kieft, K., and Anantharaman, K. (2020). Genome Diversification in Globally Distributed Novel Marine Proteobacteria is Linked to Environmental Adaptation. ISME J. 14 (8), 2060-2077. doi: 10.1038/s41396-020-0669-4

Conflict of Interest: The authors declare that the research was conducted in the absence of any commercial or financial relationships that could be construed as a potential conflict of interest.

Publisher's Note: All claims expressed in this article are solely those of the authors and do not necessarily represent those of their affiliated organizations, or those of the publisher, the editors and the reviewers. Any product that may be evaluated in this article, or claim that may be made by its manufacturer, is not guaranteed or endorsed by the publisher.

Copyright $\odot 2022$ Zhou, Chen, Zhu, Zhou, Luo, Hu, Xia and Ding. This is an openaccess article distributed under the terms of the Creative Commons Attribution License (CC BY). The use, distribution or reproduction in other forums is permitted, provided the original author(s) and the copyright owner(s) are credited and that the original publication in this journal is cited, in accordance with accepted academic practice. No use, distribution or reproduction is permitted which does not comply with these terms. 